\title{
Scattering matrix and functions of self-adjoint operators
}

\author{
Alexander Pushnitski
}

\begin{abstract}
In the scattering theory framework, we consider a pair of operators $H_{0}, H$. For a continuous function $\varphi$ vanishing at infinity, we set $\varphi_{\delta}(\cdot)=\varphi(\cdot / \delta)$ and study the spectrum of the difference $\varphi_{\delta}(H-\lambda)-\varphi_{\delta}\left(H_{0}-\lambda\right)$ for $\delta \rightarrow 0$. We prove that if $\lambda$ is in the absolutely continuous spectrum of $H_{0}$ and $H$, then the spectrum of this difference converges to a set that can be explicitly described in terms of (i) the eigenvalues of the scattering matrix $S(\lambda)$ for the pair $H_{0}, H$ and (ii) the singular values of the Hankel operator $H_{\varphi}$ with the symbol $\varphi$.
\end{abstract}

Mathematics Subject Classification (2010). Primary 47A40; Secondary 47B25.

Keywords. Scattering matrix, Hankel operators, functions of self-adjoint operators.

\section{Introduction}

1.1. Informal description of the main result. Let $H_{0}$ and $H$ be self-adjoint operators in a Hilbert space $\mathcal{H}$. We assume that $H_{0}$ and $H$ are semibounded from below and $H=H_{0}+V$ as a quadratic form sum, where $V$ is $H_{0}$-form compact. Then the difference of resolvents $(H-z I)^{-1}-\left(H_{0}-z I\right)^{-1}$ is compact for every non-real $z$ and the essential spectra of $H_{0}$ and $H$ coincide.

Let $C_{0}(\mathbb{R})$ be the set of all continuous functions $\varphi: \mathbb{R} \rightarrow \mathbb{R}$ such that $\varphi(x) \rightarrow 0$ as $|x| \rightarrow \infty$. For $\varphi \in C_{0}(\mathbb{R})$ and $\delta>0$, we denote $\varphi_{\delta}(x)=\varphi(x / \delta)$. Fix $\lambda \in \mathbb{R}$ and consider the difference

$$
A(\delta)=\varphi_{\delta}(H-\lambda)-\varphi_{\delta}\left(H_{0}-\lambda\right) .
$$

Under our assumptions it is easy to see that the operator $A(\delta)$ is compact. In this paper, we study the spectrum of $A(\delta)$ for $\delta \rightarrow+0$.

In Section 2 we make some assumptions typical for smooth scattering theory. These assumptions ensure that the (local) wave operators corresponding to the pair $H_{0}, H$ and some interval $\Delta$ in the absolutely continuous spectrum of $H_{0}$ exist and are complete. Thus, the scattering matrix $S(\lambda)$ is well defined for $\lambda \in \Delta$. For $\lambda \in \Delta$ we describe the limiting behaviour of the spectrum of $A(\delta)$ as $\delta \rightarrow+0$ explicitly in terms of 
(i) the eigenvalues of the scattering matrix $S(\lambda)$;

(ii) the singular values of the (compact) Hankel operator $H_{\varphi}$ with the symbol $\varphi$.

To give a general flavour of our result, let us consider the case $\varphi(x)=1 /\left(1+x^{2}\right)$. This case turns out to be particularly simple as the operator $H_{\varphi}$ has rank one. We prove that the spectrum of $A(\delta)$ converges (in some precise sense to be specified in Section 2) to the set

$$
\left\{ \pm \frac{1}{4}\left|s_{n}(\lambda)-1\right|: s_{n}(\lambda) \in \operatorname{spec}(S(\lambda))\right\} .
$$

We note that the link between the properties of the difference $\varphi(H)-\varphi\left(H_{0}\right)$ and the theory of Hankel operators was first exhibited in the work [11] by V. Peller. The question discussed in this paper gives another example of this link.

1.2. Connection to the Birman-Kreĭn formula. In [8], M. G. Kreŭn has proved that under some assumptions of the trace class type on the pair $H_{0}$ and $H$, for all sufficiently smooth functions $\varphi \in C_{0}(\mathbb{R})$ the operator $\varphi(H)-\varphi\left(H_{0}\right)$ belongs to the trace class and

$$
\operatorname{Tr}\left(\varphi(H)-\varphi\left(H_{0}\right)\right)=\int_{-\infty}^{\infty} \varphi^{\prime}(t) \xi(t) d t,
$$

where the function $\xi$ is known as the spectral shift function. The relation between the spectral shift function and the scattering matrix $S(\lambda)$ for the pair $H_{0}, H$ was found later in the paper [2] by M. Sh. Birman and M. G. Krel̆n (see also the previous works [10], [3], and [4]):

$$
\operatorname{det} S(\lambda)=e^{-2 \pi i \xi(\lambda)}
$$

for almost every $\lambda$ in the absolutely continuous spectrum of $H_{0}$.

Obviously, the 1.h.s. of (1.3) is the sum of the eigenvalues of $\varphi(H)-\varphi\left(H_{0}\right)$ and the 1.h.s. of (1.4) is the product of the eigenvalues of $S(\lambda)$. Thus, (1.3) and (1.4) relate the spectrum of $\varphi(H)-\varphi\left(H_{0}\right)$ to the spectrum of $S(\lambda)$. The main result of this paper gives an affirmative answer to the following

Question. Are there any other relationships between the spectrum of $\varphi(H)-\varphi\left(H_{0}\right)$ for smooth $\varphi$ and the spectrum of the scattering matrix $S(\lambda)$ for the pair $H_{0}, H$ ?

For discontinuous functions $\varphi$ the operator $A(\delta)$ may fail to be compact; see Section 6 in [8] and [7]. In this case the essential spectrum and the absolutely continuous spectrum of $A(\delta)$ can be explicitly described in terms of the spectrum of the scattering matrix; see [13], [14], and [15]. This fact is closely related to the subject of this work; it gives another relationship between the spectra of $\varphi(H)-\varphi\left(H_{0}\right)$ and $S(\lambda)$.

Acknowledgements. The author is grateful to Yu. Safarov and N. Filonov for a number of useful remarks on the text of the paper. 


\section{Main result}

2.1. Notation and assumptions. For a self-adjoint operator $A$ and a Borel set $\Lambda \subset$ $\mathbb{R}$, we denote by $E_{A}(\Lambda)$ the spectral projection of $A$ corresponding to $\Lambda$ and let $N_{A}(\Lambda)=\operatorname{rank} E_{A}(\Lambda)$. If $\Lambda=(a, b)$, we write $E_{A}(a, b), N_{A}(a, b)$ rather than $E_{A}((a, b)), N_{A}((a, b))$ in order to make our formulas more readable.

We assume that $H_{0}$ is a semi-bounded from below self-adjoint operator in a Hilbert space $\mathscr{H}$, and $V$ is another operator in $\mathscr{H}$ which is considered as the perturbation of $H_{0}$. It will be convenient to represent $V$ in a factorised form: $V=G V_{0} G$, where $G=|V|^{1 / 2}$ and $V_{0}=\operatorname{sign}(V)$. We assume that for any $\gamma<\inf \operatorname{spec}\left(H_{0}\right)$ one has

$$
\operatorname{Dom}\left(H_{0}-\gamma I\right)^{1 / 2} \subset \operatorname{Dom} G \text { and } G\left(H_{0}-\gamma I\right)^{-1 / 2} \text { is compact. }
$$

It follows that $V$ is $H_{0}$-form compact, and therefore we can define the self-adjoint operator $H$ corresponding to the form sum $H_{0}+V$ (see the "KLMN Theorem", cf. Theorem X.17 in [16]).

We fix a compact interval $\Delta \subset \mathbb{R}$ and assume that the spectrum of $H_{0}$ in $\Delta$ is purely absolutely continuous with a constant multiplicity $N \leq \infty$. More explicitly, we assume that for some auxiliary Hilbert space $\mathcal{N}, \operatorname{dim} \mathcal{N}=N$, there exists a unitary operator $\mathcal{F}$ from $\operatorname{Ran} E_{H_{0}}(\Delta)$ to $L^{2}(\Delta, \mathcal{N})$, such that $\mathcal{F}$ diagonalizes $H_{0}$ : if $f \in \operatorname{Ran} E_{H_{0}}(\Delta)$ then

$$
\left(\mathscr{F} H_{0} f\right)(\lambda)=\lambda(\mathscr{F} f)(\lambda), \quad \lambda \in \Delta .
$$

Next, we make an assumption typical for smooth scattering theory; in the terminology of [17], we assume that $G$ is strongly $H_{0}$-smooth on $\Delta$ with some exponent $\alpha \in(0,1]$. This means that the operator

$$
G_{\Delta} \stackrel{\text { def }}{=} G E_{H_{0}}(\Delta): \operatorname{Ran} E_{H_{0}}(\Delta) \rightarrow \mathscr{H}
$$

satisfies

$$
\left(\mathscr{F} G_{\Delta}^{*} \psi\right)(\lambda)=Z(\lambda) \psi, \quad \forall \psi \in \mathscr{H}, \quad \lambda \in \Delta,
$$

where $Z=Z(\lambda): \mathscr{H} \rightarrow \mathcal{N}$ is a family of compact operators obeying

$$
\|Z(\lambda)\| \leq C, \quad\left\|Z(\lambda)-Z\left(\lambda^{\prime}\right)\right\| \leq C\left|\lambda-\lambda^{\prime}\right|^{\alpha}, \quad \lambda, \lambda^{\prime} \in \Delta .
$$

The notion of strong smoothness is not unitary invariant, as it depends on the choice of the map $\mathcal{F}$. It follows from (2.3) that the operator $G_{\Delta}$ acts in $\operatorname{Ran} E_{H_{0}}(\Delta)$ according to the formula

$$
G_{\Delta} f=\int_{\Delta} Z(\lambda)^{*} F(\lambda) d \lambda, \quad F=\mathscr{F} f \in L^{2}(\Delta, \mathcal{N}) .
$$

Let us summarize our assumptions. 
Assumption 2.1. A1. $H=H_{0}+V$ (as a form sum), where $V=G V_{0} G$ satisfies (2.1).

A2. $H_{0}$ has a purely absolutely continuous spectrum with multiplicity $N$ on the interval $\Delta$.

A3. $G=|V|^{1 / 2}$ is strongly $H_{0}$-smooth on $\Delta$, i.e. (2.3) and (2.4) hold true.

2.2. Scattering theory. Recall that for a Borel set $\Lambda \subset \mathbb{R}$, the (local) wave operators are introduced by the relation

$$
W_{ \pm}\left(H, H_{0} ; \Lambda\right)=\underset{t \rightarrow \pm \infty}{\mathrm{s}-\lim _{t \rightarrow \infty}} e^{i H t} e^{-i H_{0} t} E_{H_{0}}(\Lambda) P_{H_{0}}^{(a)},
$$

provided these strong limits exist. Here and in what follows we denote by $P_{H_{0}}^{(a)}$ the orthogonal projection onto the absolutely continuous subspace of $H_{0}$. If the wave operators are complete, i.e. if the relations

$$
\operatorname{Ran} W_{+}\left(H, H_{0} ; \Lambda\right)=\operatorname{Ran} W_{-}\left(H, H_{0} ; \Lambda\right)=\operatorname{Ran}\left(E_{H}(\Lambda) P_{H}^{(a)}\right)
$$

hold true, then the (local) scattering operator is defined as

$$
\mathbf{S}=\mathbf{S}\left(H, H_{0} ; \Lambda\right)=W_{+}\left(H, H_{0} ; \Lambda\right)^{*} W_{-}\left(H, H_{0} ; \Lambda\right) .
$$

The scattering operator $\mathbf{S}$ commutes with $H_{0}$ and it is unitary on the subspace $\operatorname{Ran}\left(E_{H_{0}}(\Lambda) P_{H_{0}}^{(a)}\right)$.

For $\operatorname{Im} z \neq 0$, we set $R(z)=(H-z I)^{-1}, R_{0}(z)=\left(H_{0}-z I\right)^{-1}$. Let us define the sandwiched resolvent $T(z)$ formally by

$$
T(z)=G R_{0}(z) G, \quad \operatorname{Im} z \neq 0 ;
$$

more precisely, this means

$$
T(z)=\left(G\left(H_{0}-\gamma I\right)^{-1 / 2}\right)\left(H_{0}-\gamma I\right) R_{0}(z)\left(G\left(H_{0}-\gamma I\right)^{-1 / 2}\right)^{*}
$$

for any $\gamma<\inf \operatorname{spec}\left(H_{0}\right)$. By (2.1), the operator $T(z)$ is compact. We need the following well-known results (see e.g., Section 4.4 in [17]). Below the interior of $\Delta$ is denoted by $\operatorname{int}(\Delta)$.

Proposition 2.2. Let Assumption 2.1 hold.

(i) The operator-valued function $T(z)$ defined by (2.6) is uniformly Hölder continuous for $\operatorname{Re} z \in \operatorname{int}(\Delta), \operatorname{Im} z>0$; in particular, the limits $T(\lambda+i 0)$ exist in the operator norm and are Hölder continuous in $\lambda \in \operatorname{int}(\Delta)$. Let $\Omega \subset \operatorname{int}(\Delta)$ be the set of $\lambda$ such that the equation

$$
f+T(\lambda+i 0) V_{0} f=0
$$

has no non-trivial solutions $f \in \mathcal{H}$. Then $\Omega$ is open and $\Delta \backslash \Omega$ has the Lebesgue measure zero. The inverse operator $\left(I+T(\lambda+i 0) V_{0}\right)^{-1}, \lambda \in \Omega$, exists, is bounded and is a Hölder continuous function of $\lambda \in \Omega$.

(ii) The local wave operators $W_{ \pm}\left(H, H_{0} ; \Omega\right)$ exist and are complete. Moreover, the spectrum of $H$ in $\Omega$ is purely absolutely continuous. 
The last statement of Proposition 2.2 is usually formulated under the additional assumption $\operatorname{Ker} G=\{0\}$. Actually, this assumption is not necessary; this is verified in Lemma A.1 of [15].

Since the scattering operator $\mathbf{S}$ commutes with $H_{0}$, we have a representation

$$
\left(\mathcal{F} \mathbf{S} \mathscr{F}^{*} f\right)(\lambda)=S(\lambda) f(\lambda), \quad \text { a.e. } \lambda \in \Delta,
$$

where the operator $S(\lambda): \mathcal{N} \rightarrow \mathcal{N}$ is called the scattering matrix for the pair of operators $H_{0}, H$. The scattering matrix is a unitary operator in $\mathcal{N}$. We need the stationary representation for the scattering matrix (see [17], Chapter 7, for the details):

$$
S(\lambda)=I-2 \pi i Z(\lambda) V_{0}\left(I+T(\lambda+i 0) V_{0}\right)^{-1} Z(\lambda)^{*}, \quad \lambda \in \Omega .
$$

This representation, in particular, implies that $S(\lambda)$ is a Hölder continuous function of $\lambda \in \Omega$. Since the operator $V_{0}\left(I+T(\lambda+i 0) V_{0}\right)^{-1}$ is bounded and $Z(\lambda)$ is compact, it follows that the operator $S(\lambda)-I$ is compact. Thus, the spectrum of $S(\lambda)$ consists of eigenvalues accumulating possibly only to the point 1 . All eigenvalues of $S(\lambda)$ distinct from 1 have finite multiplicities. We denote by $\left\{s_{n}(\lambda)\right\}_{n=1}^{N}$ the eigenvalues of $S(\lambda)$, enumerated with multiplicities taken into account.

2.3. Hankel operators. Recall that the Hardy space $H^{2}\left(\mathbb{C}_{ \pm}\right) \subset L^{2}(\mathbb{R})$ is defined as the class of all functions $f$ analytic in $\mathbb{C}_{ \pm}=\{z \in \mathbb{C}: \pm \operatorname{Im} z>0\}$ and satisfying the estimate

$$
\sup _{y>0} \int_{\mathbb{R}}|f(x \pm i y)|^{2} d x<\infty .
$$

Let $P_{ \pm}$be the orthogonal projection in $L^{2}(\mathbb{R})$ onto $H^{2}\left(\mathbb{C}_{ \pm}\right)$. The explicit formula for $P_{ \pm}$is

$$
\left(P_{ \pm} f\right)(x)=\mp \frac{1}{2 \pi i} \lim _{\varepsilon \rightarrow+0} \int_{\mathbb{R}} \frac{f(y)}{x-y \pm i \varepsilon} d y .
$$

For $\varphi \in C_{0}(\mathbb{R})$ we denote by $\varphi$ the operator of multiplication by $\varphi(x)$ in $L^{2}(\mathbb{R}, d x)$ and by $H_{\varphi}$ the Hankel operator in $L^{2}(\mathbb{R})$ with the symbol $\varphi: H_{\varphi}=P_{-} \varphi P_{+}$. It is well-known [12] that the assumption $\varphi \in C_{0}(\mathbb{R})$ implies that $H_{\varphi}$ is compact. We denote by $\left\{\mu_{m}(\varphi)\right\}_{m=1}^{\infty}$ the sequence of singular values of $H_{\varphi}$ enumerated in decreasing order with multiplicities taken into account.

It is easy to check that for $\varphi(x)=1 /\left(1+x^{2}\right)$ one has

$$
H_{\varphi} f=-\frac{1}{4} v(x) \int_{\mathbb{R}} f(y) v(y) d y, \quad v(x)=\frac{1}{\sqrt{\pi}} \frac{1}{x-i} .
$$

Since $\|v\|_{L^{2}(\mathbb{R})}=1$ we see that for this choice of $\varphi$ the singular values of $H_{\varphi}$ are $\mu_{1}(\varphi)=1 / 4$ and $\mu_{m}(\varphi)=0$ for all $m \geq 2$. 
2.4. Main result. Let $\varphi \in C_{0}(\mathbb{R})$; fix $\lambda \in \Omega$ (the set $\Omega$ is defined in Proposition 2.2) and let $A(\delta)$ be as in (1.1). Let us define the set

$$
\sigma_{0}(\varphi, \lambda)=\left\{ \pm \mu_{m}(\varphi)\left|s_{n}(\lambda)-1\right|: n=1, \ldots, N, m \in \mathbb{N}\right\} \cup\{0\} .
$$

As we will see, this set is the limiting spectrum of $A(\delta)$ as $\delta \rightarrow 0$. The corresponding eigenvalue counting function is defined as

$$
N_{0}(s)=\#\left\{n=1, \ldots, N, m \in \mathbb{N}: \mu_{m}(\varphi)\left|s_{n}(\lambda)-1\right|>s\right\}, \quad s>0 .
$$

Our main result is the following.

Theorem 2.3. Let Assumption 2.1 hold true; fix $\varphi \in C_{0}(\mathbb{R})$ and $\lambda \in \Omega$. Let $A(\delta)$ be as in (1.1). Then for any $s>0, s \notin \sigma_{0}(\varphi, \lambda)$, one has

$$
N_{ \pm A(\delta)}(s, \infty) \rightarrow N_{0}(s), \quad \text { as } \delta \rightarrow+0 .
$$

It is easy to translate this statement into the more explicit language of eigenvalues. We will say that a point $v \in \sigma_{0}(\varphi, \lambda), v \neq 0$, has multiplicity $k \geq 1$ in $\sigma_{0}(\varphi, \lambda)$ if $v$ can be represented as $\pm \mu_{m}(\varphi)\left|s_{n}(\lambda)-1\right|$ for $k$ distinct choices of $n, m$. For $v \notin \sigma_{0}(\varphi, \lambda)$ we set the multiplicity of $v$ to be zero. The multiplicity of $v$ can be alternatively defined as $k=N_{0}(|v|+0)-N_{0}(|v|-0)$.

Corollary 2.4. Let $v \in \mathbb{R}, v \neq 0$ and suppose that the multiplicity of $v$ in $\sigma_{0}(\varphi, \lambda)$ is $k \geq 0$. Then for any sufficiently small $\rho>0$ there exists $\delta=\delta(\rho)$ such that for all $\delta^{\prime} \in(0, \delta]$ the operator $A\left(\delta^{\prime}\right)$ has exactly $k$ eigenvalues (counting multiplicities) in the interval $(v-\rho, v+\rho)$.

Applying Theorem 2.3 to $\varphi(x)=1 /\left(1+x^{2}\right)$ and recalling (2.9), we obtain a particularly simple relation

$$
N_{ \pm A(\delta)}(s, \infty) \rightarrow \#\left\{n=1, \ldots, N: \frac{1}{4}\left|s_{n}(\lambda)-1\right|>s\right\}, \quad \delta \rightarrow+0,
$$

for all $s>0$ such that $s \neq \frac{1}{4}\left|s_{n}(\lambda)-1\right|, n \in \mathbb{N}$.

Theorem 2.3 is proven in Sections 3 and 4. In Section 3 we introduce a model operator $A_{0}(\delta)$ (see (3.1)) such that $A_{0}(\delta)$ is unitarily equivalent to $A_{0}(1)$ for all $\delta>0$ and the spectrum of $A_{0}(1)$ is given by $\sigma_{0}(\varphi, \lambda)$. After this, we prove, roughly speaking, that $\left\|A(\delta)-A_{0}(\delta)\right\| \rightarrow 0$ as $\delta \rightarrow+0$ (see Lemma 3.2 for the precise statement); this yields (2.12). The representation (2.7) plays a key role in the proof.

2.5. Applications and extensions. Let $H_{0}=-\Delta$ in $L^{2}\left(\mathbb{R}^{d}\right)$ with $d \geq 1$. Application of the Fourier transform shows that $H_{0}$ has a purely absolutely continuous spectrum $[0, \infty)$ with multiplicity $N=2$ if $d=1$ and $N=\infty$ if $d \geq 2$.

Let $H=H_{0}+V$, where $V$ is the operator of multiplication by a function $V: \mathbb{R}^{d} \rightarrow \mathbb{R}$ which is assumed to satisfy

$$
|V(x)| \leq C(1+|x|)^{-\rho}, \quad \rho>1 .
$$


Then Assumption 2.1 is fulfilled on every compact subinterval $\Delta$ of $(0, \infty)$. Moreover, by a well-known argument involving Agmon's "bootstrap" [1] and Kato's theorem [5] on the absence of positive eigenvalues of $H$, the operator $I+T(\lambda+i 0) V_{0}$ is invertible for all $\lambda>0$ and hence $\Omega=\operatorname{int}(\Delta)$. Thus, Proposition 2.2 implies that the wave operators $W_{ \pm}\left(H, H_{0}\right)$ exist and are complete (this result was first obtained in $[6,9])$. The scattering matrix $S(\lambda)$ is a $2 \times 2$ unitary matrix if $d=1$ and a unitary operator in $L^{2}\left(\mathbb{S}^{d-1}\right)$ if $d \geq 2$. (Here $\mathbb{S}^{d-1}$ is a unit sphere in $\mathbb{R}^{d}$.) Theorem 2.3 applies to this situation.

Similar applications are possible in situations where the diagonalization of $H_{0}$ is known explicitly. For example, the perturbed Schrödinger operator with a constant magnetic field in dimension three can be considered.

Next, the construction of this paper can easily be extended to the case of operators $H_{0}, H$ which are not lower semi-bounded. Here one can follow [15] to define the sum $H_{0}+V$ in an appropriate way and to prove that $A(\delta)$ is compact for $\varphi \in C_{0}(\mathbb{R})$. The rest of the construction remains the same.

\section{Proof of Theorem 2.3}

3.1. The model operator. Below we assume without loss of generality that $\lambda=0$ and let $a>0$ be such that the interval $[-a, a]$ belongs to the set $\Omega$. For $\delta>0$, let us define the model operator $A_{0}(\delta)$ in $L^{2}(\mathbb{R}, \mathcal{N})=L^{2}(\mathbb{R}) \otimes \mathcal{N}$ by

$$
A_{0}(\delta)=H_{\varphi_{\delta}} \otimes(S(0)-I)+H_{\varphi_{\delta}}^{*} \otimes\left(S(0)^{*}-I\right) .
$$

By definition, the operator $A_{0}(\delta)$ is self-adjoint. Since both $H_{\varphi_{\delta}}$ and $S(0)-I$ are compact, the operator $A_{0}(\delta)$ is also compact.

For $\delta>0$, let $U(\delta)$ in $L^{2}(\mathbb{R}, \mathcal{N})$ be the unitary scaling operator

$$
(U(\delta) f)(x)=\delta^{-1 / 2} f(x / \delta), \quad x \in \mathbb{R} .
$$

It is straightforward to see that

$$
A_{0}(\delta)=U(\delta) A_{0}(1) U(\delta)^{*}
$$

in particular, the spectrum and the eigenvalue counting function of $A_{0}(\delta)$ are independent of $\delta$.

Lemma 3.1. For all $\delta>0$, the spectrum of $A_{0}(\delta)$ coincides with the set $\sigma_{0}(\varphi, 0)$ defined in (2.10). For any $s>0, s \notin \sigma_{0}(\varphi, 0)$, one has

$$
N_{ \pm A_{0}(\delta)}(s, \infty)=N_{0}(s),
$$

with $N_{0}(s)$ defined in (2.11). 
Proof. By (3.2), it suffices to consider the case $\delta=1$. Let the eigenvalues of $S(0)$ be $s_{n}(0)=e^{i \theta_{n}}, \theta_{n} \in[0,2 \pi)$, and let $\alpha_{n}=\left(\theta_{n}+\pi\right) / 2$. Using the spectral decomposition of $S(0)$, one represents the operator $A_{0}(\delta)$ as the orthogonal sum of the operators

$$
\left(s_{n}(0)-1\right) H_{\varphi}+\left(\overline{s_{n}(0)}-1\right) H_{\varphi}^{*}=\left|s_{n}(0)-1\right|\left(e^{i \alpha_{n}} P_{-} \varphi P_{+}+e^{-i \alpha_{n}} P_{+} \varphi P_{-}\right)
$$

in $L^{2}(\mathbb{R})$. With respect to the orthogonal sum decomposition $L^{2}(\mathbb{R})=H^{2}\left(\mathbb{C}_{-}\right) \oplus$ $H^{2}\left(\mathbb{C}_{+}\right)$we have

$$
e^{i \alpha_{n}} P_{-} \varphi P_{+}+e^{-i \alpha_{n}} P_{+} \varphi P_{-}=\left(\begin{array}{cc}
0 & e^{i \alpha_{n}} P_{-} \varphi P_{+} \\
e^{-i \alpha_{n}} P_{+} \varphi P_{-} & 0
\end{array}\right) .
$$

A simple argument shows that if $M$ is a compact operator with the singular values $\left\{\mu_{m}\right\}_{m=1}^{\infty}$, then the spectrum of $\left(\begin{array}{cc}0 & M \\ M^{*} & 0\end{array}\right)$ consists of the eigenvalues $\left\{ \pm \mu_{m}\right\}_{m=1}^{\infty}$. Thus, we get that the spectrum of the operator in the r.h.s. of (3.4) consists of the eigenvalues $\left\{ \pm \mu_{m}(\varphi)\right\}_{m=1}^{\infty}$. Combining this with (3.3), we obtain the required statement.

3.2. The strategy of proof. Let $\Pi_{\mathcal{N}}, \Pi_{\mathscr{H}}$ be the restriction operators:

$$
\Pi_{\mathcal{N}}: L^{2}(\mathbb{R}, \mathcal{N}) \rightarrow L^{2}((-a, a), \mathcal{N}) \quad \text { and } \quad \Pi_{\mathscr{H}}: L^{2}(\mathbb{R}, \mathscr{H}) \rightarrow L^{2}((-a, a), \mathscr{H}) .
$$

Recall that $\mathcal{F}$ is defined in Section 2.1 (see (2.2)); we set

$$
\mathscr{F}_{a}=\mathscr{F}_{H_{0}}(-a, a): \mathscr{H} \rightarrow L^{2}((-a, a), \mathcal{N}) .
$$

Consider the partial isometry

$$
Q: L^{2}(\mathbb{R}, \mathcal{N}) \rightarrow \mathscr{H}, \quad Q=\mathscr{F}_{a}^{*} \Pi_{\mathcal{N}}
$$

It is clear that $\|Q\|=1$. In Section 4 we prove

Lemma 3.2. As $\delta \rightarrow+0$, one has

$$
\begin{aligned}
\left\|A(\delta)-Q A_{0}(\delta) Q^{*}\right\| & \rightarrow 0, \\
\left\|\left(Q^{*} Q-I\right) A_{0}(\delta)\right\| & \rightarrow 0, \\
\left\|\left(Q Q^{*}-I\right) A(\delta)\right\| & \rightarrow 0 .
\end{aligned}
$$

Given Lemma 3.2, it is not difficult to complete the proof of Theorem 2.3.

Proof of Theorem 2.3. 1. First note that from (3.5)-(3.7) it follows that

$$
\left\|A_{0}(\delta)-Q^{*} A(\delta) Q\right\| \rightarrow 0, \quad \delta \rightarrow+0 .
$$

Indeed,

$$
A_{0}(\delta)=\left(I-Q^{*} Q\right) A_{0}(\delta)+Q^{*} Q A_{0}(\delta)\left(I-Q^{*} Q\right)+Q^{*} Q A_{0}(\delta) Q^{*} Q,
$$


and therefore, using the fact that $\|Q\|=1$,

$$
\begin{aligned}
\left\|A_{0}(\delta)-Q^{*} A(\delta) Q\right\| & \leq\left\|\left(I-Q^{*} Q\right) A_{0}(\delta)\right\|+\left\|Q^{*} Q A_{0}(\delta)\left(I-Q^{*} Q\right)\right\| \\
& +\left\|Q^{*}\left(Q A_{0}(\delta) Q^{*}-A(\delta)\right) Q\right\| \\
& \leq 2\left\|\left(I-Q^{*} Q\right) A_{0}(\delta)\right\|+\left\|Q A_{0}(\delta) Q^{*}-A(\delta)\right\| \rightarrow 0
\end{aligned}
$$

as $\delta \rightarrow 0$.

2. Fix $s>0, s \notin \sigma_{0}(\varphi, 0)$ and let $\varepsilon \in(0, s), \varepsilon<1$. Using (3.5)-(3.8), let us choose $\delta_{0}>0$ such that for all $\delta \in\left(0, \delta_{0}\right)$ we have

$$
\begin{gathered}
\left\|\left(Q^{*} Q-I\right) A_{0}(\delta)\right\|<s \varepsilon, \quad\left\|\left(Q Q^{*}-I\right) A(\delta)\right\|<s \varepsilon, \\
\left\|A_{0}(\delta)-Q^{*} A(\delta) Q\right\|<\varepsilon, \quad\left\|A(\delta)-Q A_{0}(\delta) Q^{*}\right\|<\varepsilon .
\end{gathered}
$$

Let us prove that from (3.9), (3.10) it follows that

$$
\begin{aligned}
N_{A_{0}(\delta)}\left(\frac{s-\varepsilon}{1+\varepsilon}, \infty\right) & \geq N_{A(\delta)}(s, \infty), \\
N_{A(\delta)}\left(\frac{s-\varepsilon}{1+\varepsilon}, \infty\right) & \geq N_{A_{0}(\delta)}(s, \infty)
\end{aligned}
$$

for all $\delta \in\left(0, \delta_{0}\right)$. We prove (3.11); the relation (3.12) is proven in the same way. Let $f \in \operatorname{Ran} E_{A(\delta)}(s, \infty),\|f\|=1$; then $f=A(\delta) g,\|g\| \leq 1 / s$. Using (3.9), we get

$$
\left\|\left(Q Q^{*}-I\right) f\right\|=\left\|\left(Q Q^{*}-I\right) A(\delta) g\right\|<s \varepsilon / s=\varepsilon .
$$

It follows that

$$
\left|\left(\left(Q Q^{*}-I\right) f, f\right)\right|<\varepsilon,
$$

or equivalently,

$$
\left|\left\|Q^{*} f\right\|^{2}-1\right|<\varepsilon
$$

Since $\varepsilon<1$, we obtain $\left\|Q^{*} f\right\| \neq 0$, and also

$$
\left\|Q^{*} f\right\|^{2}<1+\varepsilon
$$

Further, by the definition of $f$ we have $(A(\delta) f, f) \geq s$ and so, using (3.10), we get

$$
\left(A_{0}(\delta) Q^{*} f, Q^{*} f\right)=(A(\delta) f, f)+\left(\left(Q A_{0}(\delta) Q^{*}-A(\delta)\right) f, f\right)>s-\varepsilon .
$$

Combining this with (3.13), we obtain

$$
\frac{\left(A_{0}(\delta) Q^{*} f, Q^{*} f\right)}{\left\|Q^{*} f\right\|^{2}}>\frac{s-\varepsilon}{1+\varepsilon} .
$$

Since we have already seen that $Q^{*} f \neq 0$, we get that $Q^{*}$ maps $\operatorname{Ran} E_{A(\delta)}(s, \infty)$ onto a subspace $L, \operatorname{dim} L=N_{A(\delta)}(s, \infty)$ and for all $h \in L$ we have

$$
\left(A_{0}(\delta) h, h\right)>\frac{s-\varepsilon}{1+\varepsilon}\|h\|^{2} .
$$


By the min-max principle, we get (3.11).

3. Recall that by Lemma 3.1 the spectrum of $A_{0}(\delta)$ is independent of $\delta$ and coincides with $\sigma_{0}(\varphi, 0)$. Since by assumption $s \notin \sigma_{0}(\varphi, 0)$, the interval $\left[\frac{s-\varepsilon}{1+\varepsilon}, s\right]$ contains no eigenvalues of $A_{0}(\delta)$ if $\varepsilon$ is sufficiently small; then the 1.h.s. of (3.11) equals $N_{A_{0}(\delta)}(s, \infty)$. Thus, (3.11) is equivalent to

$$
N_{A_{0}(\delta)}(s, \infty) \geq N_{A(\delta)}(s, \infty) .
$$

Let us prove the opposite inequality. Denote $s^{\prime}=s(1+\varepsilon)+\varepsilon$; then (3.9), (3.10) hold true with $s^{\prime}$ in place of $s$ and therefore (3.12) also holds true with $s^{\prime}$ in place of $s$ :

$$
N_{A(\delta)}\left(\frac{s^{\prime}-\varepsilon}{1+\varepsilon}, \infty\right) \geq N_{A_{0}(\delta)}\left(s^{\prime}, \infty\right) .
$$

This can be rewritten as

$$
N_{A(\delta)}(s, \infty) \geq N_{A_{0}(\delta)}(s(1+\varepsilon)+\varepsilon, \infty) .
$$

Again, if $\varepsilon$ is chosen sufficiently small then the r.h.s. in the last inequality equals $N_{A_{0}(\delta)}(s, \infty)$. Thus, we get $N_{A(\delta)}(s, \infty) \geq N_{A_{0}(\delta)}(s, \infty)$; combining this with (3.14) yields

$$
N_{A(\delta)}(s, \infty)=N_{A_{0}(\delta)}(s, \infty)
$$

for all sufficiently small $\delta$. In the same way, one proves that

$$
N_{-A(\delta)}(s, \infty)=N_{-A_{0}(\delta)}(s, \infty)
$$

for all $s \notin \sigma_{0}(\varphi, 0)$ and all sufficiently small $\delta$. Thus, we arrive at (2.12).

\section{Proof of Lemma 3.2}

4.1. Preliminaries. Here we prove the relations (3.5), (3.6) and (3.7). The proof of (3.6) and (3.7) is very straightforward, while the proof of (3.5) requires more detailed analysis. We will repeatedly use the following well-known fact. Let $M_{n}$ be a sequence of bounded operators such that $M_{n} \rightarrow 0$ strongly as $n \rightarrow \infty$. Then for any compact operator $K$, one has $\left\|M_{n} K\right\| \rightarrow 0$ as $n \rightarrow \infty$. In particular, if we also have $M_{n}^{*} \rightarrow 0$ strongly, then $\left\|K M_{n}\right\|=\left\|M_{n}^{*} K^{*}\right\| \rightarrow 0$ as $n \rightarrow \infty$.

4.2. The proof of (3.6) and (3.7) . Let us prove (3.6). We have

$$
Q^{*} Q=\left(\mathcal{F}_{a}^{*} \Pi_{\mathcal{N}}\right)^{*} \mathscr{F}_{a}^{*} \Pi_{\mathcal{N}}=\Pi_{\mathcal{N}}{ }^{*} \mathscr{F}_{a} \mathscr{F}_{a}^{*} \Pi_{\mathcal{N}}=\Pi_{\mathcal{N}^{*}} \Pi_{\mathcal{N}}=\chi_{(-a, a)},
$$

where $\chi_{(-a, a)}$ is the operator of multiplication by the characteristic function of the interval $(-a, a)$ in $L^{2}(\mathbb{R}, \mathcal{N})$. It follows that

$$
\left\|\left(Q^{*} Q-I\right) A_{0}(\delta)\right\|=\left\|\left(\chi_{(-a, a)}-I\right) U(\delta) A_{0}(1) U(\delta)^{*}\right\| .
$$


It is straightforward to see that the operator $\left(\chi_{(-a, a)}-I\right) U(\delta)$ converges to zero strongly as $\delta \rightarrow+0$. Since $A_{0}(1)$ is a compact operator, we obtain (3.6).

Let us prove (3.7). By the definition of $Q$, we have

$$
Q Q^{*}=\mathscr{F}_{a}^{*} \Pi_{\mathcal{N}} \Pi_{\mathcal{N}}^{*} \mathcal{F}_{a}=\mathscr{F}_{a}^{*} \mathcal{F}_{a}=E_{H_{0}}(-a, a) .
$$

Thus, we need to prove that

$$
\left\|\left(E_{H_{0}}(-a, a)-I\right) A(\delta)\right\| \rightarrow 0, \quad \delta \rightarrow 0 .
$$

Let $\zeta \in C(\mathbb{R})$ be such that $\zeta(x)=1$ for $|x| \geq a$ and $\zeta(x)=0$ for $|x| \leq a / 2$. Clearly, it suffices to prove that

$$
\left\|\zeta\left(H_{0}\right) A(\delta)\right\| \rightarrow 0, \quad \delta \rightarrow 0 .
$$

We have

$$
\zeta\left(H_{0}\right) A(\delta)=\zeta(H) \varphi_{\delta}(H)-\zeta\left(H_{0}\right) \varphi_{\delta}\left(H_{0}\right)+\left(\zeta\left(H_{0}\right)-\zeta(H)\right) \varphi_{\delta}(H) .
$$

Consider separately the three terms in the r.h.s. of (4.3). Since $\varphi(x) \rightarrow 0$ as $|x| \rightarrow 0$, we have $\left\|\zeta(H) \varphi_{\delta}(H)\right\| \rightarrow 0$ and $\left\|\zeta\left(H_{0}\right) \varphi_{\delta}\left(H_{0}\right)\right\| \rightarrow 0$ as $\delta \rightarrow+0$. Next, denoting $\tilde{\zeta}(x)=1-\zeta(x)$, we have $\tilde{\zeta} \in C_{0}(\mathbb{R})$. It follows that the operator

$$
\zeta\left(H_{0}\right)-\zeta(H)=\tilde{\zeta}(H)-\tilde{\zeta}\left(H_{0}\right)
$$

is compact. By our assumptions we have $0 \in \Omega$ ( $\Omega$ is defined in Proposition 2.2), and therefore 0 is not an eigenvalue of $H$. It follows that $\varphi_{\delta}(H)$ converges to zero strongly as $\delta \rightarrow 0$. Thus, we get

$$
\left\|\varphi_{\delta}(H)\left(\zeta\left(H_{0}\right)-\zeta(H)\right)\right\| \rightarrow 0
$$

as $\delta \rightarrow 0$, and therefore the last term in the r.h.s. of (4.3) converges to zero in the operator norm. Thus, (3.7) is proven.

In the rest of this section, we prove (3.5).

4.3. Notation. Denote $Y(z)=V_{0}\left(I+T(z) V_{0}\right)^{-1}, \operatorname{Im} z>0$; by Proposition 2.2, the limit $Y(x+i 0)$ exists for all $x \in[-a, a]$ and is a Hölder continuous function of $x$ in the operator norm. Recall that the operators $Z(x), x \in[-a, a]$, are defined in Section 2.1 (see (2.3), (2.4)). Let us define the operators

$$
\mathcal{Z}, \mathcal{Z}_{0}: L^{2}(\mathbb{R}, \mathscr{H}) \rightarrow L^{2}(\mathbb{R}, \mathcal{N}) \quad \text { and } \quad y, y_{0}: L^{2}(\mathbb{R}, \mathcal{H}) \rightarrow L^{2}(\mathbb{R}, \mathcal{H})
$$

by

$$
\left(\mathcal{Z}_{0} f\right)(x)=Z(0) f(x), \quad\left(y_{0} f\right)(x)=Y(+i 0) f(x), \quad x \in \mathbb{R},
$$

and

$$
(Z) f)(x)=\left\{\begin{array}{ll}
Z(x) f(x) & |x| \leq a, \\
0 & |x|>a,
\end{array} \quad(y f)(x)= \begin{cases}Y(x+i 0) f(x) & |x| \leq a, \\
0 & |x|>a .\end{cases}\right.
$$

We will also need the operator valued versions of the Hardy projections $P_{ \pm}$(see (2.8)). Denote by $\mathcal{P}_{\mathscr{H}, \pm}$ the operators in $L^{2}(\mathbb{R}, \mathscr{H})=L^{2}(\mathbb{R}) \otimes \mathscr{H}$ defined by $\mathcal{P}_{\mathscr{H}, \pm}=$ $P_{ \pm} \otimes I_{\mathscr{H}}$. Similarly, $\mathcal{P}_{\mathcal{N}, \pm}$ are the operators $P_{ \pm} \otimes I_{\mathcal{N}}$ in $L^{2}(\mathbb{R}, \mathcal{N})=L^{2}(\mathbb{R}) \otimes \mathcal{N}$. 
4.4. Two formulas. Here e give formulas for the operator $Q A_{0}(\delta) Q^{*}$ and the operator $E_{H_{0}}(-a, a) A(\delta) E_{H_{0}}(-a, a)$ in terms of the operators $\mathcal{Z}, Z_{0}, \mathcal{Y}, \mathcal{Y}_{0}$ introduced above.

Lemma 4.1. Let $\varphi$ be compactly supported. Then for all sufficiently small $\delta>0$, we have

$$
Q A_{0}(\delta) Q^{*}=4 \pi \operatorname{Im}\left(\widetilde{F}_{a}^{*} \Pi_{\mathcal{N}} \mathcal{P}_{\mathcal{N},-} Z_{0} y_{0} Z_{0}^{*} \varphi_{\delta} \mathcal{P}_{\mathcal{N},+} \Pi_{\mathcal{N}}^{*} \widetilde{F}_{a}\right),
$$

and

$$
\begin{aligned}
E_{H_{0}}(-a, a) A(\delta) E_{H_{0}}(-a, a) & \\
& =4 \pi \operatorname{Im}\left(\mathcal{F}_{a}^{*} \Pi_{\mathcal{N}} \mathcal{Z} \mathcal{P}_{\mathcal{H},-} y_{\varphi_{\delta}} \mathcal{P}_{\mathscr{H},+} \mathcal{Z}^{*} \Pi_{\mathcal{N}^{*}}^{*} \mathcal{F}_{a}\right) .
\end{aligned}
$$

Proof. 1. Let us prove (4.4). By the stationary representation (2.7) for the scattering matrix, we have

$$
S(0)=I-2 \pi i Z(0) Y(+i 0) Z(0)^{*} .
$$

By the definition (3.1) of $A_{0}(\delta)$, we get

$$
\begin{aligned}
A_{0}(\delta) & =2 \operatorname{Re}\left(P_{-} \varphi_{\delta} P_{+} \otimes(S(0)-I)\right)=4 \pi \operatorname{Im}\left(P_{-} \varphi_{\delta} P_{+} \otimes Z(0) Y(+i 0) Z(0)^{*}\right) \\
& =4 \pi \operatorname{Im}\left(\mathcal{P}_{\mathcal{N},-} \mathcal{Z}_{0} y_{0} \mathcal{Z}_{0}^{*} \varphi_{\delta} \mathcal{P}_{\mathcal{N},+}\right),
\end{aligned}
$$

and (4.4) follows.

2. We will use the resolvent identity in the form

$$
R(z)-R_{0}(z)=-\left(G R_{0}(\bar{z})\right)^{*} Y(z) G R_{0}(z), \quad \operatorname{Im} z>0 .
$$

Let us recall the derivation of (4.6) (see e.g. [17, Section 1.9]). Iterating the usual resolvent identity, we get

$$
\begin{aligned}
R(z)-R_{0}(z) & =-R(z) V R_{0}(z)=-R_{0}(z) V R_{0}(z)+R_{0}(z) V R(z) V R_{0}(z) \\
& =-R_{0}(z) G V_{0}\left(I-G R(z) G V_{0}\right) G R_{0}(z) .
\end{aligned}
$$

We also have the identity

$$
\left(I-G R(z) G V_{0}\right)\left(I+G R_{0}(z) G V_{0}\right)=I,
$$

which can be verified by expanding and using (4.7). Substituting (4.9) into (4.8) and using the notation $Y(z)$, we obtain (4.6).

3. Let us prove (4.5). Let $\delta>0$ be sufficiently small so that $\operatorname{supp} \varphi_{\delta} \subset[-a, a]$. First recall a version of Stone's formula:

$$
\left(\varphi_{\delta}(H) f, f\right)=\frac{1}{\pi} \lim _{\varepsilon \rightarrow+0} \operatorname{Im} \int_{-a}^{a}(R(x+i \varepsilon) f, f) \varphi_{\delta}(x) d x,
$$


for any $f \in \mathscr{H}$. Using this formula, a similar formula for $\varphi_{\delta}\left(H_{0}\right)$ and the resolvent identity (4.6), we get

$(A(\delta) f, f)=-\frac{1}{\pi} \lim _{\varepsilon \rightarrow+0} \operatorname{Im} \int_{-a}^{a}\left(Y(x+i \varepsilon) G R_{0}(x+i \varepsilon) f, G R_{0}(x-i \varepsilon) f\right) \varphi_{\delta}(x) d x$,

for any $f \in \mathscr{H}$.

Next, let $f \in \operatorname{Ran} E_{H_{0}}(-a, a)$ and $F=\mathscr{F}_{a} f$. From (2.5) we obtain for any $\operatorname{Im} z \neq 0$

$$
G R_{0}(z) f=\int_{-a}^{a} \frac{Z(t)^{*} F(t)}{t-z} d t .
$$

Combining (4.10) and (4.11), we obtain

$$
\begin{gathered}
(A(\delta) f, f)=4 \pi \lim _{\varepsilon \rightarrow+0} \operatorname{Im} \int_{-a}^{a} d x \int_{-a}^{a} d t \int_{-a}^{a} d s(M(x, t, s) F(t), F(s)), \\
M(x, t, s)=Z(s) \frac{1}{2 \pi i} \frac{1}{s-x-i \varepsilon} Y(x+i \varepsilon) \varphi_{\delta}(x)\left(-\frac{1}{2 \pi i}\right) \frac{1}{x-t+i \varepsilon} Z(t)^{*} .
\end{gathered}
$$

Recalling formula (2.8) for $P_{ \pm}$, we obtain the required identity (4.5).

\subsection{Compactness lemma}

\section{Lemma 4.2. The operators}

$$
L^{ \pm}=\Pi_{\mathcal{N}}\left(\mathcal{Z} \mathcal{P}_{\mathcal{H}, \pm}-\mathcal{P}_{\mathcal{N}, \pm} \mathcal{Z}\right) \Pi_{\mathscr{H}}^{*}: L^{2}((-a, a), \mathscr{H}) \rightarrow L^{2}((-a, a), \mathcal{N})
$$

are compact.

Proof. Clearly, it suffices to prove that for any $\varepsilon>0$ the operator $L^{ \pm}$can be represented as $L^{ \pm}=L_{\varepsilon}^{ \pm}+\widetilde{L}_{\varepsilon}^{ \pm}$, where $L_{\varepsilon}^{ \pm}$is compact and $\left\|\widetilde{L}_{\varepsilon}^{ \pm}\right\| \leq \varepsilon$.

1. Let us prove that for any $\varepsilon>0$ one can find an operator valued polynomial $Z_{\varepsilon}(x)$ with coefficients being finite rank operators from $\mathscr{H}$ to $\mathcal{N}$ and such that $\left\|Z_{\varepsilon}(x)-Z(x)\right\| \leq \varepsilon$ for all $x \in[-a, a]$. Let $P_{n, \mathscr{H}}$ be a sequence of orthogonal projections in $\mathscr{H}$ of finite rank which converges strongly to the identity operator $I_{\mathscr{H}}$. Let $P_{n, \mathcal{N}}$ be a similar sequence for the space $\mathcal{N}$. For each $x \in[-a, a]$, by compactness of $Z(x)$ we have

$$
\left\|Z(x)\left(P_{n, \mathscr{H}}-I_{\mathscr{H}}\right)\right\| \rightarrow 0, \quad\left\|\left(P_{n, \mathcal{N}}-I_{\mathcal{N}}\right) Z(x)\right\| \rightarrow 0,
$$

and therefore, by the compactness of the interval $[-a, a]$, the above convergence holds true uniformly over $x \in[-a, a]$. It follows that for a sufficiently large $n$ we have

$$
\left\|P_{n, \mathcal{N}} Z(x) P_{n, \mathscr{H}}-Z(x)\right\| \leq \varepsilon / 2, \quad x \in[-a, a] .
$$

The operator $P_{n, \mathcal{N}} Z(x) P_{n, \mathscr{H}}$ can be thought of as a matrix with respect to some bases in $\operatorname{Ran} P_{n, \mathcal{N}}$ and $\operatorname{Ran} P_{n, \mathscr{H}}$; the elements of this matrix are continuous functions 
in $x$. By the Weierstrass approximation theorem, the elements of this matrix can be approximated by polynomials uniformly on $[-a, a]$. This yields the required approximation $Z_{\varepsilon}(x)$ of $Z(x)$.

2. For $Z_{\varepsilon}$ as above, let us write $Z=Z_{\varepsilon}+\widetilde{Z}_{\varepsilon}$, with $\left\|\widetilde{Z}_{\varepsilon}(x)\right\| \leq \varepsilon$ for all $x \in[-a, a]$. This gives rise to a decomposition $L^{ \pm}=L_{\varepsilon}^{ \pm}+\widetilde{L}_{\varepsilon}^{ \pm}$with

$$
\left\|\tilde{L}_{\varepsilon}^{ \pm}\right\|=\left\|\Pi_{\mathcal{N}}\left(\tilde{Z}_{\varepsilon} \mathcal{P}_{\mathscr{H}, \pm}-\mathcal{P}_{\mathcal{N}, \pm} \tilde{\mathcal{Z}}_{\varepsilon}\right) \Pi_{\mathscr{H}}^{*}\right\| \leq 2\left\|\tilde{\mathcal{Z}}_{\varepsilon}\right\| \leq 2 \varepsilon .
$$

It suffices to prove that $L_{\varepsilon}^{ \pm}$is compact. The operator $L_{\varepsilon}^{ \pm}$is an integral operator with the kernel

$$
L_{\varepsilon}^{ \pm}(x, y)=\mp \frac{1}{2 \pi i} \frac{Z_{\varepsilon}(x)-Z_{\varepsilon}(y)}{x-y} .
$$

This is a smooth matrix valued kernel, and therefore $L_{\varepsilon}^{ \pm}$is compact.

4.6. Proof of (3.5). 1. First let us prove (3.5) for compactly supported $\varphi$. By (4.1), it suffices to prove that

$$
\left\|E_{H_{0}}(-a, a) A(\delta) E_{H_{0}}(-a, a)-Q A_{0}(\delta) Q^{*}\right\| \rightarrow 0, \quad \delta \rightarrow+0 .
$$

Since $\varphi$ is compactly supported, we can apply Lemma 4.1 to represent the operators in (4.13) in terms of $\mathcal{Z}, \mathcal{Y}$, etc. Thus, we see that (4.13) will follow from

$$
\left\|\Pi_{\mathcal{N}} \mathcal{Z} \mathcal{P}_{\mathcal{H},-} y_{\varphi_{\delta}} \mathcal{P}_{\mathscr{H},+} \mathcal{Z}^{*} \Pi_{\mathcal{N}}^{*}-\Pi_{\mathcal{N}} \mathcal{P}_{\mathcal{N},-} \mathcal{Z}_{0} y_{0} \mathcal{Z}_{0}^{*} \varphi_{\delta} \mathcal{P}_{\mathcal{N},+} \Pi_{\mathcal{N}}^{*}\right\| \rightarrow 0
$$

$\delta \rightarrow+0$. Below we prove (4.14).

2 . Note that $\varphi_{\delta}$ converges to zero strongly as $\delta \rightarrow+0$. Next, if $\delta$ is sufficiently small so that $\operatorname{supp} \varphi_{\delta} \subset[-a, a]$, we have

$$
\Pi_{\mathcal{N}}\left(\mathcal{Z} \mathcal{P}_{\mathscr{H},-}-\mathcal{P}_{\mathcal{N},-} \mathcal{Z}\right) \varphi_{\delta}=\Pi_{\mathcal{N}}\left(\mathcal{Z} \mathcal{P}_{\mathscr{H},-}-\mathcal{P}_{\mathcal{N},-} \mathcal{Z}\right) \Pi_{\mathcal{H}}^{*} \Pi_{\mathscr{H}} \varphi_{\delta}=L^{-} \Pi_{\mathscr{H}} \varphi_{\delta},
$$

where $L^{-}$is defined in (4.12). Thus by Lemma 4.2,

$$
\left\|\Pi_{\mathcal{N}}\left(\mathcal{Z} \mathcal{P}_{\mathcal{H},-}-\mathcal{P}_{\mathcal{N},-} \mathcal{Z}\right) \varphi_{\delta}\right\| \rightarrow 0, \quad \delta \rightarrow+0 .
$$

Writing a similar relation for $L^{+}$instead of $L^{-}$and taking adjoints, one obtains

$$
\left\|\varphi_{\delta}\left(\mathcal{P}_{\mathcal{H},+} \mathfrak{Z}^{*}-\mathfrak{Z}^{*} \mathcal{P}_{\mathcal{N},+}\right) \Pi_{\mathcal{N}}^{*}\right\| \rightarrow 0, \quad \delta \rightarrow+0 .
$$

Combining (4.15) and (4.16) and using the commutation $\varphi_{\delta} y=y_{\varphi_{\delta}}$, we obtain

$$
\left\|\Pi_{\mathcal{N}} \mathcal{Z} \mathcal{P}_{\mathcal{H},-} y_{\varphi_{\delta}} \mathcal{P}_{\mathcal{H},+} \mathcal{Z}^{*} \Pi_{\mathcal{N}}^{*}-\Pi_{\mathcal{N}} \mathcal{P}_{\mathcal{N},-} \mathcal{Z} y_{\varphi_{\delta}} \mathcal{Z}^{*} \mathcal{P}_{\mathcal{N},+} \Pi_{\mathcal{N}}^{*}\right\| \rightarrow 0,
$$

$\delta \rightarrow+0$. Recall that by Proposition 2.2 the operator $Y(x+i 0)$ is continuous in $x \in[-a, a]$. Using this fact and the continuity of $Z(x)$ in $x$ we get

$$
\left\|\mathcal{Z} y_{\varphi_{\delta}} Z^{*}-Z_{0} y_{0} \varphi_{\delta} Z_{0}^{*}\right\| \rightarrow 0, \quad \delta \rightarrow+0 .
$$


Combining the last relation with (4.17) and using the commutation $\varphi_{\delta} Z_{0}=Z_{0} \varphi_{\delta}$, we arrive at (4.14).

3. It remains to extend (3.5) from compactly supported functions to general $\varphi \in C_{0}(\mathbb{R})$. For $\varphi \in C_{0}(\mathbb{R})$ and any given $\varepsilon>0$ let us approximate $\varphi$ by a compactly supported function $\tilde{\varphi}$ such that $\|\varphi-\tilde{\varphi}\|_{C(\mathbb{R})} \leq \varepsilon$. Let $\tilde{A}(\delta), \widetilde{A_{0}}(\delta)$ be the operators $A(\delta), A_{0}(\delta)$ corresponding to $\tilde{\varphi}$. Then by steps 1 and 2 above we have $\left\|\widetilde{A}(\delta)-Q \widetilde{A}_{0}(\delta) Q^{*}\right\| \rightarrow 0$ as $\delta \rightarrow+0$. Next, directly from the definition of $A(\delta)$ and $A_{0}(\delta)$ we obtain

$$
\begin{aligned}
\|A(\delta)-\tilde{A}(\delta)\| & \leq 2\|\varphi-\tilde{\varphi}\|_{C(\mathbb{R})} \leq 2 \varepsilon \\
\left\|A_{0}(\delta)-\tilde{A}_{0}(\delta)\right\| & \leq 4\|\varphi-\tilde{\varphi}\|_{C(\mathbb{R})} \leq 4 \varepsilon
\end{aligned}
$$

It follows that

$$
\limsup _{\delta \rightarrow+0}\left\|A(\delta)-Q A_{0}(\delta) Q^{*}\right\| \leq 6 \varepsilon .
$$

Since $\varepsilon>0$ is arbitrary, we get (3.5).

\section{References}

[1] S. Agmon, Spectral properties of Schrödinger operators and scattering theory. Ann. Scuola Norm. Sup. Pisa IV 2 (1975), 151-218. MR 0397194 Zbl 0315.47007

[2] M. Sh. Birman and M. G. Kreĭn, On the theory of wave operators and scattering operators. Dokl. Akad. Nauk SSSR 144 (1962), 475-478. English transl.: Soviet Math. Dokl. 3 (1962), 740-744. MR 0139007 Zbl 0196.45004

[3] V. S. Buslaev and L. D. Faddeev, Formulas for traces for a singular Sturm-Liouville differential operator. Dokl. Akad. Nauk SSSR 132 (1960), 13-16. English traslation in Soviet Math. Dokl. 1 (1960), 451-454. MR 0120417 Zbl 0129.06501

[4] V. S. Buslaev, The trace formulae and certain asymptotic estimates of the kernel of the resolvent for the Schrödinger operator in three-dimensional space. Probl. Mat. Fiz. 1 (1966), 82-101. In Russian. MR 0205110 Zbl 0203.13403

[5] T. Kato, Growth properties of solutions of the reduced wave equation with a variable coefficient. Commun. Pure Appl. Math. 12 (1959), 403-425. MR 0108633 Zbl 0091.09502

[6] T. Kato, Some results on potential scattering. In Proceedings of the international conference on functional analysis and related topics, Tokyo, April, 1969. Mathematical Society of Japan by University of Tokyo Press, Tokyo, 1970, 206-215. MR 0268713 Zbl 0207.45303

[7] V. Kostrykin and K. Makarov, On Kreŭn's example, Proc. Amer. Math. Soc. 136 (2008), 2067-2071. MR 2383512 Zbl 1143.47019

[8] M. G. Kreı̆n, On the trace formula in perturbation theory. Mat. Sb. 33(75) (1953), 597626. MR $0060742 \mathrm{Zbl} 0052.12303$

[9] S. T. Kuroda, Scattering theory for differential operators I: Operator Theory and II: Self-adjoint elliptic operators. J. Math. Soc. Japan 25 (1973), 75-104; ibid. 222-234. MR 48:4779 (I) Zbl 0245.47006 (I); MR 48:4780 (II) Zbl 0252.47007 (II) 
[10] I. M. Lifšic, On degenerate regular perturbations. II: Quasicontinuous and continuous spectrum, Akad. Nauk SSSR. Zhurnal Eksper. Teoret. Fiz. 17 (1947), 1076-1089. MR 0023451

[11] V. Peller, Hankel operators in perturbation theory of unitary and self-adjoint operators, Funct. Anal. Appl. 19 (1985), 111-123. MR 0800919 Zbl 0587.47016

[12] V. Peller, Hankel operators and their applications. Springer Verlag, New York, 2003. MR 1949210 Zbl 1030.47002

[13] A. Pushnitski, The scattering matrix and the differences of spectral projections. Bull. Lond. Math. Soc. 40 (2008), 227-238. MR 2414782 Zbl 1145.47009

[14] A. Pushnitski, Spectral theory of discontinuous functions of self-adjoint operators: essential spectrum. Integral Equations Oper. Theory 68 (2010), 75-99. MR 2677889 Zbl 1198.47016

[15] A. Pushnitski and D. Yafaev, Spectral theory of discontinuous functions of self-adjoint operators and scattering theory. J. Funct. Anal. 259 (2010), 1950-1973. MR 2671117 Zbl 05782189

[16] M. Reed and B. Simon, Methods of modern mathematical physics. II. Fourier Analysis, Self-adjointness. Academic Press, New York etc., 1975. MR 0493420 Zbl 0308.47002

[17] D. R. Yafaev, Mathematical scattering theory. General theory. Amer. Math. Soc., Providence (RI), 1992. MR 1180965 Zbl 0761.47001

Received August 6, 2010

Alexander Pushnitski, Department of Mathematics, King's College London, Strand, London, WC2R 2LS, U.K.

E-mail: alexander.pushnitski@kcl.ac.uk 